University of Brighton

\title{
Multi-professional approaches to children's foot health: a qualitative study
}

\author{
Lisa Hodgson $^{1}$, Anita Williams ${ }^{2}$, Chris Nester $^{2}$, Stewart C Morrison ${ }^{1}$
}

1. School of Health Sciences, University of Brighton 2. School of Health and Society, University of Salford

\section{Correspondence: Lisa Hodgson L.Hodgson@Brighton.ac.uk Twitter: @Lisa_Hodgson_}

Purpose: Health professionals have an important influence on children's foot health throughout childhood. The foot is a common site of injury and parents regularly seek access to health services. Given this, it is imperative that the knowledge and practices utilised by health professionals are consistent and build upon the best available evidence. The purpose of this work was to understand more about the mutual behaviours and opinions in how health professionals find and share information that is both relevant to their practice, as well as informing the advice they share with parents.

Methods: A qualitative design using semi-structured, one-to-one, telephone interviews with allied health professionals was adopted. All transcripts were transcribed verbatim and thematic analysis was used. All transcripts were coded to generate meaning, identify patterns, the frequency of common phrasing and develop themes from the data.

Results: Eight interviews were conducted with physiotherapists, podiatrists and orthotists. Five themes were identified relating to (1) Engaging with research (2) Experience over evidence (3) Influence of children's footwear companies (4) Dr Google- the new experts (5) Influence of referral pathways.

Conclusions: The findings indicate that health professionals adopt a number of strategies to inform their professional knowledge such as engaging with peer knowledge when evidence-based research could be uncertain. There were barriers that health professionals experienced which made it difficult to apply evidence into a clinical setting e.g. information could be complex, varied, profession specific, out of date. There were also challenges in the way children were referred into paediatric foot services, often resulting in inefficient utilisation of services and resources, and a representation of different advice and information given to parents, which could impact on the health professional and parent engagement during consultations.

Implications: The findings from this work support strategies to improve engagement, networking, awareness of health professional disciplines and knowledge sharing to inform referral pathways between health professionals. This work also supports the need to progress education initiatives to support greater health literacy in parents.

Acknowledgments: We would like to thank Charlotte Growcott for her support and contribution to the work looking at multi-professional approaches to children's foot health.

Funding acknowledgment: The Dr. William M Scholl Unit of Podiatric Development

To learn more about the project or to follow our progress please use the following links

Great Foundations Website $@$ @rtFoundations Twitter page

Facebook page 